\title{
The shape of vortices in quasi-geostrophic turbulence
}

\author{
By J. N. REINAUD ${ }^{1}$, D. G. DRITSCHEL ${ }^{1}$ \\ AND C. R. KOUDELLA ${ }^{2}$ \\ ${ }^{1}$ Mathematical Institute, University of St Andrews, North Haugh, St Andrews KY16 9SS, UK \\ ${ }^{2}$ Department of Applied Mathematics and Theoretical Physics, University of Cambridge, \\ Centre for Mathematical Sciences, Wilberforce Road, Cambridge CB3 0WA, UK
}

(Received 2 November 2001 and in revised form 15 July 2002)

The present work discusses the most commonly occurring shape of the coherent vortical structures in rapidly rotating stably stratified turbulence, under the quasigeostrophic approximation. In decaying turbulence, these vortices - coherent regions of the materially-invariant potential vorticity-dominate the flow evolution, and indeed the flow evolution is governed by their interactions. An analysis of several exceptionally high-resolution simulations of quasi-geostrophic turbulence is performed. The results indicate that the population of vortices exhibits a mean height-to-width aspect ratio less than unity, in fact close to 0.8 .

This finding is justified here by a simple model, in which vortices are taken to be ellipsoids of uniform potential vorticity. The model focuses on steady ellipsoids within a uniform background strain flow. This background flow approximates the effects of surrounding vortices in a turbulent flow on a given vortex. It is argued that the vortices which are able to withstand the highest levels of strain are those most likely to be found in the actual turbulent flow. Our calculations confirm that the optimal height-to-width aspect ratio is close to 0.8 for a wide range of background straining flows.

\section{Introduction}

The quasi-geostrophic model contains the main dynamical features of rapidly rotating stably stratified flows commonly found in the atmosphere and oceans. The flow is fully described by the advection of a Lagrangian invariant, the 'potential vorticity', together with a simple inversion relation giving the velocity field in terms of the distribution of potential vorticity. In this model, the dynamics of turbulence reduces principally to the interaction between coherent masses of potential vorticity, or 'vortices'. Over the past two decades, there have been many studies of quasigeostrophic turbulence, mainly computational, which have addressed both the spectral and physical-space (vortical) properties of the flow. These studies, in part, sought to test a theory of isotropic turbulence proposed by Charney (1971). This theory states that spectral properties, and by implication flow properties, should have the same statistical variation in all directions, though in a rescaled coordinate system in which the height is stretched by the ratio of the buoyancy $N$ to Coriolis $f$ frequencies (both taken to be spatially uniform). This theory is the basis for the idea that the typical ratio of vertical to horizontal scales is $O(f / N)$ in large-scale atmospheric and oceanic motions. 
The computational studies, however, have not fully supported this theory. McWilliams, Weiss \& Yavneh (1994a) found that the potential vorticity tends to condense into tall approximately columnar structures or vortices. On the other hand, in Dritschel, de la Torre Juárez \& Ambaum (1999b), more isotropic vortices were found to emerge, even from initial conditions consisting of columnar vortices. This is in agreement with the stability analysis of Dritschel \& de la Torre Juárez (1996), who showed that tall columnar vortices are generally unstable and break down into flatter vortices.

The difference between these simulation results can be traced back to the initial conditions used. Dritschel \& Macaskill (2000) showed that a small to moderate number of initially columnar vortices may remain columnar in a periodic domain, despite the tall-column instability. However, upon doubling the domain width in each direction (and likewise doubling the horizontal resolution), the columnar vortices may break down into three-dimensional structures. In a wide enough domain, all columns destabilize and the flow remains fully three-dimensional for long times. It is remarkable that the initial diameter of vortices for which the tall-column instability is inhibited can be as small as $5 \%$ of the domain width. Although the initial conditions used by McWilliams et al. (1994a) were of smaller scale and of a different (random) character, numerical diffusion acts strongly at early times to produce vortical structures of such a width, and these subsequently begin to condense into two columnar structures, which are stabilized by the periodic effects.

McWilliams et al. (1994a) noted that vortices emerge with a roughly spherical shape at early times. Further analyses of this same simulation (McWilliams et al. 1994b; McWilliams et al. 1999) indicated first that the flow develops a spectral anisotropy and furthermore that, in physical space, coherent vortices develop a mean height-to-width aspect ratio of $0.83 \pm 0.03$, i.e. an oblate spheroid in the rescaled reference frame proposed by Charney (1971). The latter result was obtained from a moderate sample of vortices identified by a census procedure which excludes highly deformed structures. Moreover, according to McWilliams et al., numerical diffusion has an influence on their solution. It is therefore of interest to re-examine the shape of vortices found in highly resolved simulations, in which the vortex population is large enough to provide well-converged statistics.

We focus on what we consider to be generic initial conditions, consisting of a large number of initially isotropic vortices which are much smaller than the domain width to avoid the effects of periodicity. Our primary goal is to understand what determines the mean shape of vortices in three-dimensional quasi-geostrophic turbulence. Recent extraordinarily large computations have been conducted by Koudella, Dritschel \& McMullen (2002) using a fast parallel extension of the contour-advective semiLagrangian (CASL) algorithm, introduced in Dritschel \& Ambaum (1997). The analysis presented below confirms that the mean height-to-width aspect ratio of vortices in three different simulations is significantly less than unity, statistically centred on a value of 0.8 in each case.

The present work justifies this result by showing that flat vortices are favoured in quasi-geostrophic turbulence. Such vortices are found to be most capable of withstanding the background strain flow arising from the surrounding vortices in the turbulent flow. We model this background flow in an idealized way, retaining only the leading-order effects of surrounding vortices on a given vortex. To that end, we investigate steady ellipsoids of potential vorticity embedded in the flow created by a single distant vortex arbitrarily positioned in space. Ellipsoids of potential vorticity have frequently been used in the literature as a simple yet pertinent model of the 
coherent structures in quasi-geostrophic flows, see for example Meacham (1992), Meacham et al. (1994), Hashimoto, Shimonishi \& Miyazaki (1999), Miyazaki, Ueno \& Shimonishi (1999) and Miyazaki, Furuichi \& Takahashi (2001). We use here a formalism derived from the reformulation of the equations of motion of an ellipsoid in McKiver \& Dritschel (2003). This formalism enables us to find steady states for a broad range of the parameters characterizing the vortex shape and the background strain flow. The key idea of this work is to find the aspect ratio for the ellipsoid corresponding to the maximum strain below which steady states can be found. Vortices with this shape would be able to withstand the effects of the background strain better than would vortices with any other shape. We argue that, in turbulence, vortices with this shape will survive the longest, and therefore will be seen most frequently.

The paper is organized as follows. Section 2 introduces the quasi-geostrophic model and describes the results of several numerical simulations performed using the CASL algorithm. Section 3 reviews the ellipsoidal model and outlines the numerical approach taken. Results are then presented for the maximum strain below which a steady state can be found, for a fixed height-to-width aspect ratio of the ellipsoid. It is concluded in $\S 4$ that vortices with an aspect ratio less than unity should be statistically predominant in quasi-geostrophic turbulence, consistent with the numerical simulation results.

\section{The numerical experiment}

\subsection{Mathematical formulation}

The present work uses the quasi-geostrophic (QG) model, in which the potential vorticity (PV) is materially conserved (in the absence of diabatic and dissipative effects). Under the assumption that both the rotational and the buoyancy frequencies $f$ and $N$ are constant, and after multiplying the vertical coordinate by the ratio $N / f$, the governing equations are

$$
\begin{gathered}
\frac{\mathrm{D} q}{\mathrm{D} t}=0, \\
\Delta \psi=q, \\
u=-\frac{\partial \psi}{\partial y}, \quad v=\frac{\partial \psi}{\partial x},
\end{gathered}
$$

where $q$ is the $\mathrm{PV}, \mathrm{D} \cdot / \mathrm{D} t$ is the material derivative, and $\Delta$ is the Laplacian (see e.g. Gill (1982) for details of the model). Although Laplace's operator is isotropic, a natural anisotropy occurs in the flow owing to the lack of vertical advection-the flow is layerwise two-dimensional. The vertical direction is therefore special, and we cannot a priori expect flow isotropy.

The CASL algorithm uses a hybrid Lagrangian/Eulerian solution method for these equations. PV is enclosed within horizontal contours which are explicitly advected, and dissipated when they reach a small prescribed 'surgical' scale (to allow for topological reconnections). This approach takes advantage of the stability of Lagrangian methods and allows us to use time steps that are related to the inherently slow time scale present in the flow, measured by the PV rather than by stability criteria (e.g. CFL) which would force us to use a much smaller time step, see Cottet (1996) and Dritschel et al. $(1999 b)$. On the other hand, the method benefits from the accuracy of an Eulerian spectral approach when inverting the Poisson equation (2) for the streamfunction. A complete discussion of the method can be found in Dritschel \& Ambaum (1997), and in Koudella et al. (2002) for the parallel algorithm. 


$\begin{array}{clrll}\text { Resolution } & p & V_{\max } / V_{\min } & F & n_{\text {tot }} \\ 512^{3} & 2 & 25 & 0.1 & 1000 \\ 640^{3} & 0.9 & 1000 & 0.1 & 2000 \\ 1024^{3} & - & 1 & 0.08 & 5000\end{array}$

TABLE 1. Description of the initial conditions for the three CASL simulations.

\subsection{Numerical results}

Three simulations of QG turbulence have been performed in a triply periodic cubic domain of equal dimensions (after the $N / f$ scaling of $z$ discussed above). The starting conditions consist of uniform-PV spherical vortices, with an equal number having $q=+Q$ and $q=-Q$, with $Q=4 \pi$ so that a unit of time corresponds to a "vortex rotation period'. (The period of rotation for a column of uniform PV is $4 \pi /|Q|$, and for a sphere it is $6 \pi /|Q|$.) The three simulations differ in their resolution $\left(512^{3}, 640^{3}\right.$, and $1024^{3}$ ) and especially in the initial distribution of vortex sizes, to ensure that the results are not dependent on the initial conditions chosen. The vortex volumes $V$ were selected from a number density distribution $n(V) \propto V^{-p}$, with a specified ratio of the maximum to minimum volume $V_{\max } / V_{\min }$, fraction $F$ of the domain volume occupied by the vortices, and total number of vortices $n_{t o t}=\int_{V_{\min }}^{V_{\max }} n(V) \mathrm{d} V$. The vortices are randomly positioned in space, starting with the largest vortices, while taking care that they do not overlap one another. The values of the parameters used are given in table 1 . Note that in the $1024^{3}$ case, the value of $p$ is immaterial since all the vortices have the same size initially. The initial condition for the $640^{3}$ simulation is shown in figure 1.

As for the numerical parameters, each simulation used the same time step $\Delta t=$ 0.025 , a surgical scale of one-tenth of the basic grid scale, and a fine-grid/coarse-grid ratio of 2 (the latter is used in converting the PV contours to gridded values). The values of these parameters are those recommended in Dritschel \& Ambaum (1997). Judging from comparative studies (cf. Dritschel, Polvani \& Mohebalhojeh 1999a), they result in solutions which are much more accurate than those obtainable with standard numerical methods (e.g. pseudospectral) using the same grid sizes.

The results of each simulation were analysed over the same time window, from $t=250$ to 350 , and at intervals of 2 time units. By this time, the flow has relaxed to a slowly evolving state of widely separated vortices, which only occasionally strongly interact-for example, figure 2 shows the PV distribution in the $640^{3}$ simulation at $t=300$.

The analysis consists first of identifying all contiguous regions of PV, hereinafter called 'vortices'. (This simple identification procedure differs substantially from that introduced by McWilliams (1990) and used in subsequent works.) Patches of PV in adjacent vertical layers are considered contiguous if they are found to overlap one another. Holes inside patches are not considered part of the vortex, e.g. when volume is calculated. Next, the volumes and other properties of the vortices are calculated. The volumes $V$ are calculated by summing the areas of all contours (negative areas correspond to holes) belonging to the vortex and then multiplying by the layer thickness (or vertical grid size). These areas are computed from discrete contour integrals, using the standard formula $A=\frac{1}{2} \oint x \mathrm{~d} y-y \mathrm{~d} x$. Then the centre $\boldsymbol{X}$ of each vortex is computed from $X=V^{-1} \int \boldsymbol{x} \mathrm{d} V$ (taking into account periodicity), and 


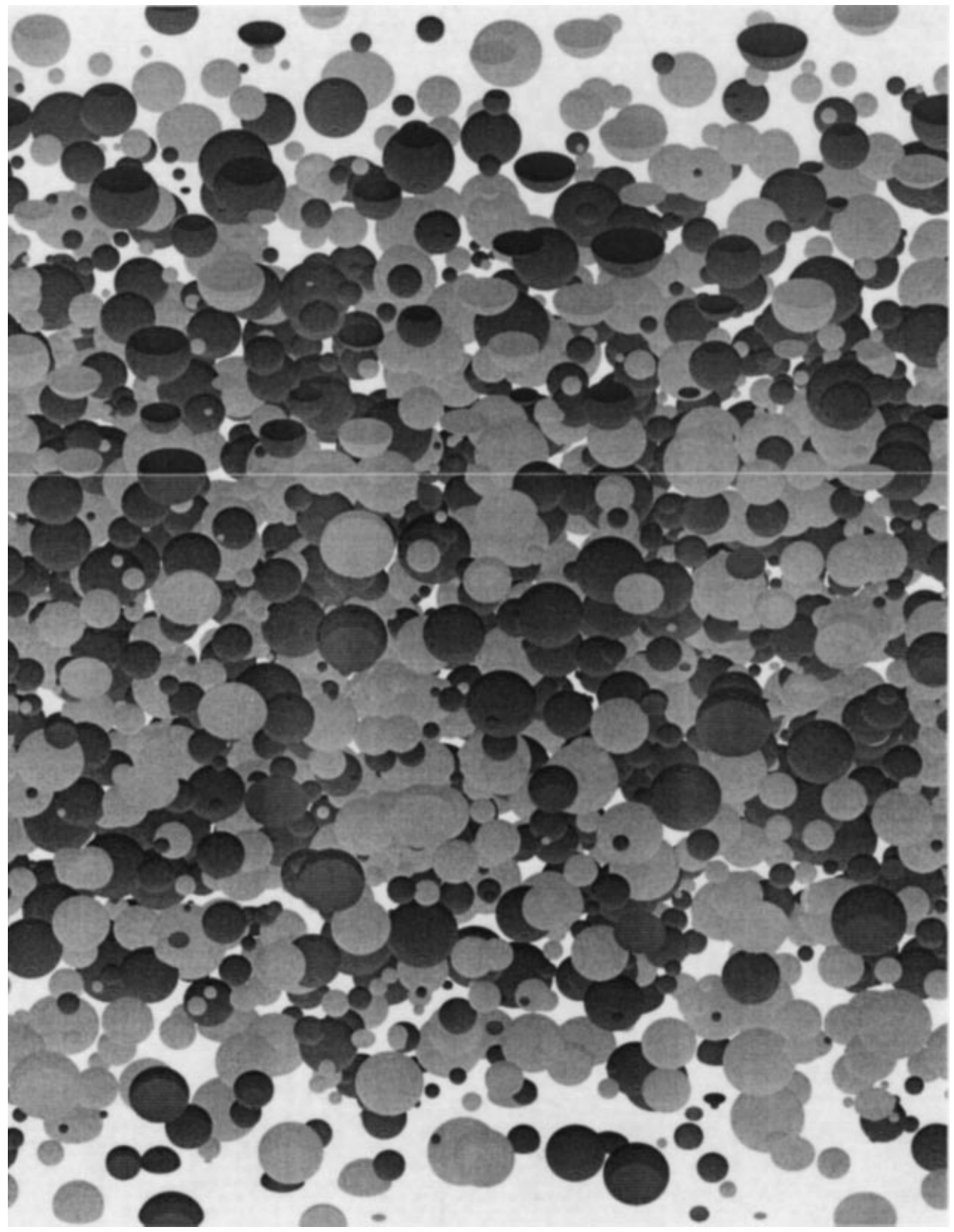

Figure 1. A snapshot of the initial conditions for the $640^{3}$ simulation. The PV distribution is viewed orthographically, at an angle of $60^{\circ}$ from the vertical, and in the plane $y=0$. From this view, we can see the top and front faces of the domain. Positive PV is rendered a slightly darker shade of grey than negative PV.

finally the vortex shape is computed by fitting an ellipsoid having the same 'second moments' (volume integrals of $\hat{x}^{2}, \hat{x} \hat{y}, \hat{x} \hat{z}, \hat{y}^{2}, \hat{y} \hat{z}$, and $\hat{z}^{2}$, where $\left.\hat{\boldsymbol{x}}=(\hat{x}, \hat{y}, \hat{z}) \equiv \boldsymbol{x}-\boldsymbol{X}\right)$.

The principle diagnostic of interest here is the vortex height-to-width aspect ratio, $h / r$. This is computed from the above basic diagnostics as follows. We first compute 


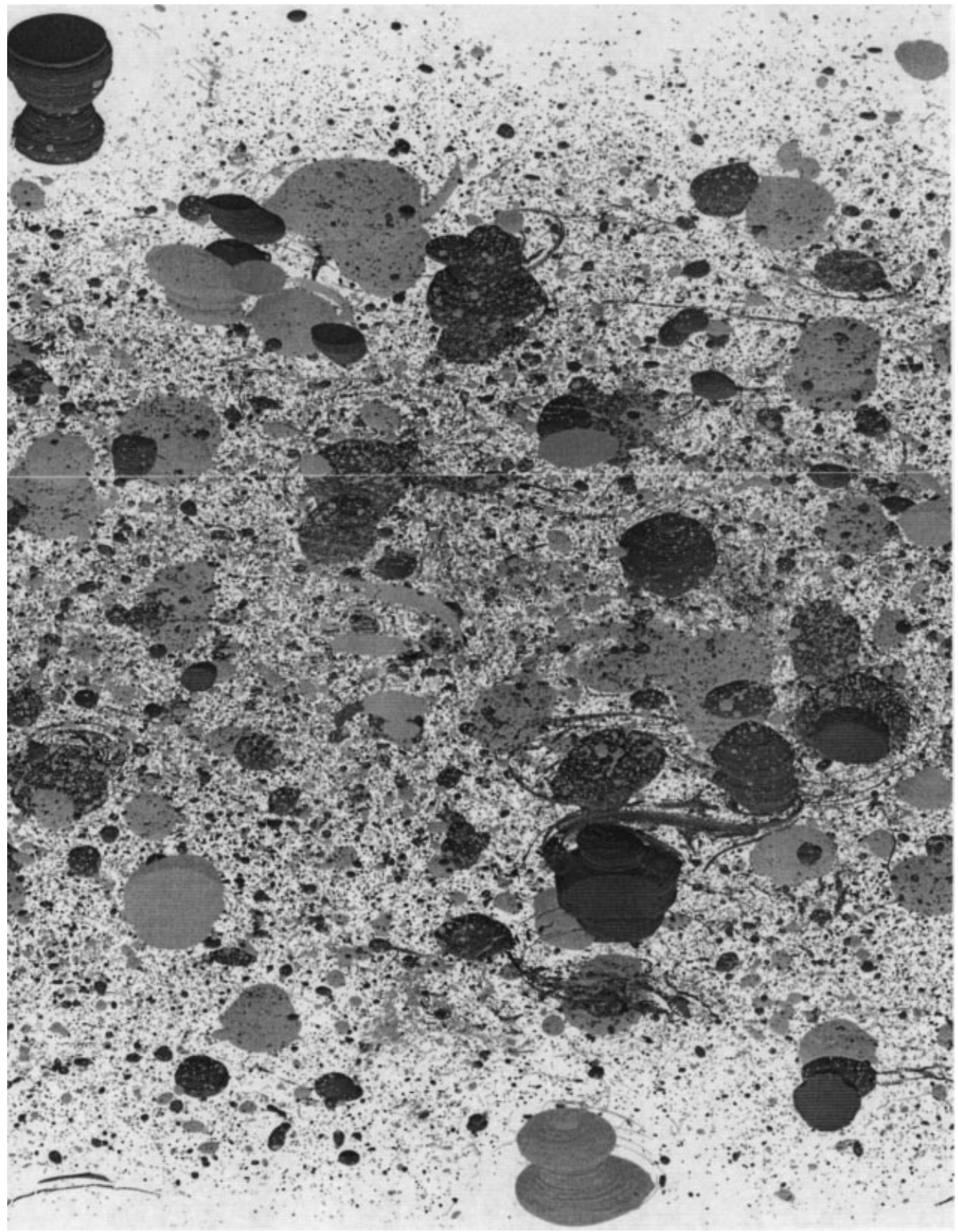

Figure 2. A snapshot of QG turbulence at $t=300$ from the $640^{3}$ simulation, rendered as in figure 1.

$h$ from $h=\sqrt{5 M_{z z} / V}$, where $M_{z z}$ is the volume integral of $\hat{z}^{2}$ over the vortex -for an ellipsoid in standard position, this reduces to the half-height of the vortex. Then, the mean horizontal radius $r$ is computed from the volume constraint, $V=\frac{4}{3} \pi r^{2} h$. Combining these formulae, we have $h / r=\left(\frac{4}{3} \pi\right)^{1 / 2}\left(5 M_{z z}\right)^{3 / 4} V^{-5 / 4}$. Again, this reduces to the correct expression for an ellipsoid, even when the ellipsoid is tilted with respect 


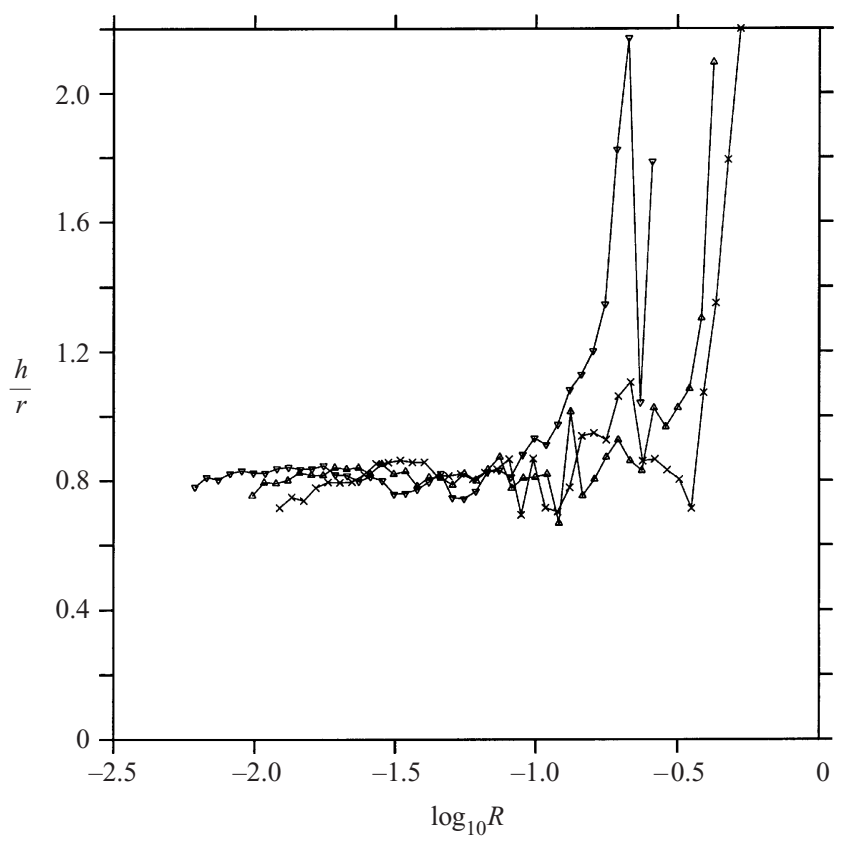

FIGURE 3. Mean height-to-width aspect ratio $\overline{h / r}$ versus $\log _{10} R$ in the CASL simulations at resolutions $\times, 512^{3} ; \boldsymbol{\Lambda}, 640^{3} ; \boldsymbol{\nabla}, 1024^{3}$.

to the vertical (then the value of $h$ does not change, since the vortex must still occupy the same height range because of the lack of vertical motion).

After computing $h / r$ for all vortices, we form an average height-to-width aspect ratio $\overline{h / r}$ over selected ranges of vortex volumes, or bins. Specifically, the logarithm (base 10) of the mean vortex radius $R \equiv(3 V / 4 \pi)^{1 / 3}$ is chopped into 40 bins of equal length, between the grid size and the radius of the largest vortex found in the time period sampled. In each bin, the values of $h / r$ are accumulated and then averaged once the sample size in each bin is determined. It is noteworthy that there are many more small vortices than there are large ones, so the sample size is marginal for large vortices (and therefore the statistics are not reliable there). However, the sample size for the small vortices is large, as indicated below.

The results of the three simulations are presented in figure 3 . The three simulations all give a mean aspect ratio of approximately 0.8 for all but the largest vortices in the population. The results are insensitive to the time period sampled (we have also examined the statistics at 150 time units later), although the statistics for the largest vortices are probably not reliable, owing to the small number of such vortices. On the other hand, the sample size for small vortices is more than ample: the overall sample sizes are 285759 vortices for the $512^{3}$ case, 655838 vortices for the $640^{3}$ case, and 2551114 vortices for the $1024^{3}$ case. The upturn towards $\overline{h / r}=1$ for the larger vortices does appear to be significant - it is observed in all three simulations as well as in different sampling periods.

To judge the significance of these results better, we have computed the probability density distribution of $h / r$ for the three simulations over several ranges of vortex radii $R$. This is done by dividing the range $0<h / r<2.5$ into 50 equal-width bins and counting the number of vortices falling into each over the time period sampled. The total sample size is then used to normalize the bin counts to give the probability 

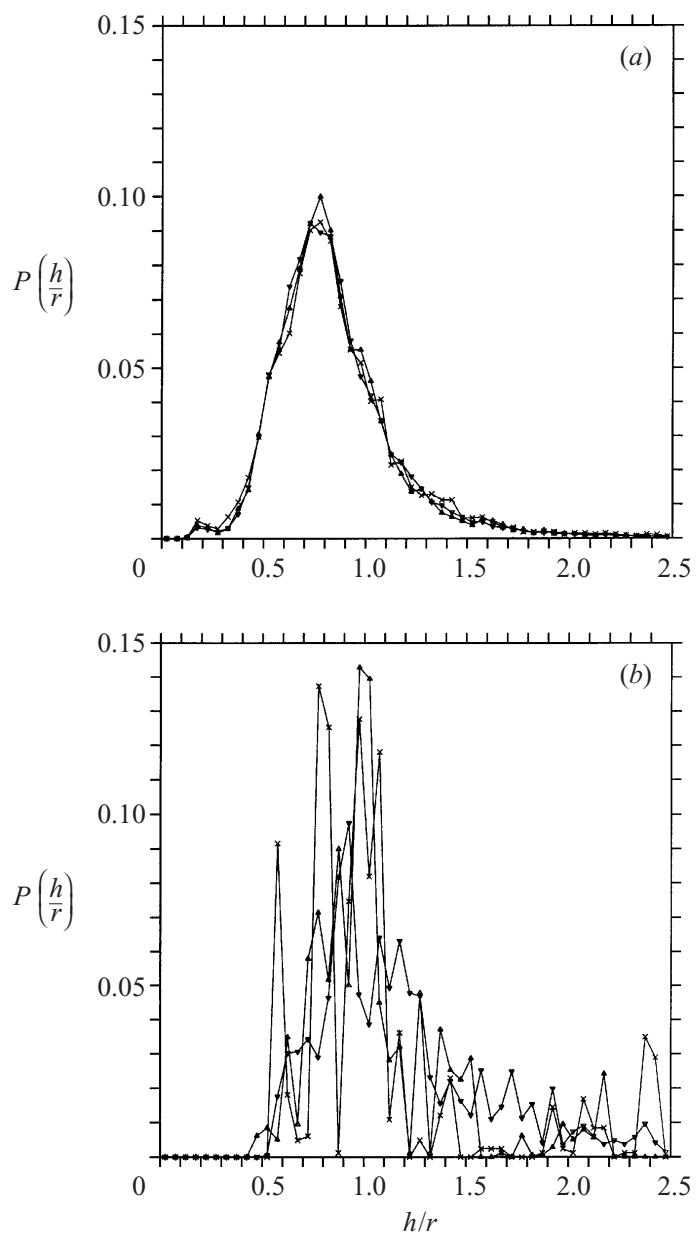

Figure 4. Probability density $P(h / r)$ versus $h / r$ for the three simulations (using the same symbols as in the previous figure), and $(a)$ for vortices with radii $R$ exceeding two grid lengths; $(b)$ for the largest vortices (having a volume at least a tenth of the largest vortex volume).

density $P(h / r)$. The results for the three simulations are presented in figure $4(a)$ for all vortices having a radius of at least two grid lengths, and in figure $4(b)$ for the upper tenth (in vortex volume) largest vortices, $R_{\max } / 10^{1 / 3}<R<R_{\max }$, where $R_{\max }$ is the maximum vortex radius over the entire sampling period. In figure $4(a)$, it is remarkable how well the data from the three simulations collapse onto one another, and exhibit a clearly defined peak and mean around $h / r=0.8$. The overall means and r.m.s. differences, together with the sample sizes, are given in table 2 . The results are in very close agreement, and clearly indicate that vortices with $h / r<1$ are dominant. Approximately $80 \%$ of the vortices have $h / r<1$. Moreover, fewer than $10 \%$ of the vortices have $h / r<0.5$, and only about $1 \%$ of the vortices have $h / r>2$. Flat vortices are therefore not common and tall vortices are decidedly rare. The results for the largest vortices, see figure $4(b)$, are much less robust owing to the much smaller sample sizes (see table 3). Although there is evidence that the largest vortices have a mean aspect ratio of order unity or slightly greater, it is premature to draw any firm conclusions. If we exclude these large vortices from the original distribution shown 


$\begin{array}{ccccccc}\text { Resolution } & \overline{h / r} & (h / r-\overline{h / r})_{r m s} & \text { Oblate fraction } & \text { Flat fraction } & \text { Tall fraction } & \text { Sample size } \\ 512^{3} & 0.8505 & 0.3980 & 0.7767 & 0.1016 & 0.0193 & 45663 \\ 640^{3} & 0.8319 & 0.3498 & 0.8013 & 0.0895 & 0.0129 & 105113 \\ 1024^{3} & 0.8352 & 0.3498 & 0.7922 & 0.0854 & 0.0132 & 462293\end{array}$

TABLE 2. Statistics for all vortices with $R>$ twice the grid size. 'Oblate fraction', 'Flat fraction' and 'Tall fraction' refer to the fraction of vortices having $h / r<1, h / r<0.5$ and $h / r>2$, respectively.

\begin{tabular}{ccccccc}
\hline Resolution & $\overline{h / r}$ & $(h / r-\overline{h / r})_{r m s}$ & Oblate fraction & Flat fraction & Tall fraction & Sample size \\
$512^{3}$ & 1.0804 & 0.5351 & 0.5957 & 0.0000 & 0.1118 & 830 \\
$640^{3}$ & 1.0366 & 0.3480 & 0.6356 & 0.0140 & 0.0427 & 1778 \\
$1024^{3}$ & 1.1851 & 0.4783 & 0.4264 & 0.0000 & 0.0716 & 9895
\end{tabular}

TABLE 3. Statistics for vortices having a volume at least one-tenth of the largest vortex volume.

\begin{tabular}{ccccccc}
\hline Resolution & $\overline{h / r}$ & $(h / r-\overline{h / r})_{r m s}$ & Oblate fraction & Flat fraction & Tall fraction & Sample size \\
$512^{3}$ & 0.8462 & 0.3937 & 0.7800 & 0.1035 & 0.0176 & 44833 \\
$640^{3}$ & 0.8284 & 0.3488 & 0.8041 & 0.0908 & 0.0124 & 103335 \\
$1024^{3}$ & 0.8275 & 0.3424 & 0.8003 & 0.0873 & 0.0119 & 452398
\end{tabular}

TABLE 4. Statistics for all vortices with $R>$ twice the grid size but excluding the largest vortices analysed.

in figure 4(a), there is no significant change in the form of $P(h / r)$, nor in the basic statistics (see table 4). The upshot is that, for all but perhaps the largest vortices, there is a strong tendency for vortices to have an aspect ratio of less than unity.

\section{The steady states}

We next present a simple model that appears to explain the numerical simulation results above.

\subsection{Formalism and numerical approach}

Following the formalism described in McKiver \& Dritschel (2003), we investigate steady or equilibrium ellipsoids of uniform PV within both a background horizontal strain and a vertical shear. In general, the background flow changes in time, but here we adopt a steadily translating and rotating frame of reference and assume steady values for the background strain and shear.

The key aspects of the analytical and numerical procedures are now presented. Let $\mathscr{A}$ be the symmetric $3 \times 3$-matrix in terms of which the equation of the ellipsoid reads

$$
\boldsymbol{x} \mathscr{A} \boldsymbol{x}^{T}=1,
$$

and $a, b$ and $c$ be the axis half-lengths. Let $\mathscr{B}=\mathscr{A}^{-1}$. Let $\mathscr{S}$ be the velocity gradient matrix $\nabla \boldsymbol{u}$, and let us assume that the flow is linear, $\boldsymbol{u}=\mathscr{S}_{\boldsymbol{x}}$. Note that $\mathscr{S}=\mathscr{S}_{b}+\mathscr{S}_{v}$ itself is the sum of the background and the ellipsoid contributions. The temporal evolution of the ellipsoid is obtained by taking a time derivative of (4) and using 
$\mathrm{d} \mathscr{A} \mathscr{B} / \mathrm{d} t=0$; the result is

$$
\frac{\mathrm{d} \mathscr{B}}{\mathrm{d} t}=\mathscr{B} \mathscr{S}^{T}+\mathscr{S} \mathscr{B},
$$

where the superscript $T$ denotes transpose.

In the present case, we set the PV of the ellipsoid to 1. Following Meacham (1994), $\mathscr{S}_{v}$ at the boundary of the ellipsoid is given by

$$
\mathscr{S}_{v}=\mathscr{L} \mathscr{M} \mathscr{F} \mathscr{M}^{T},
$$

where

$$
\mathscr{F}=\frac{a b c}{3}\left(\begin{array}{ccc}
R_{D}\left(b^{2}, c^{2}, a^{2}\right) & 0 & 0 \\
0 & R_{D}\left(c^{2}, a^{2}, b^{2}\right) & 0 \\
0 & 0 & R_{D}\left(a^{2}, b^{2}, c^{2}\right)
\end{array}\right) \text {, }
$$

with

$$
R_{D}(\alpha, \delta, \epsilon)=\frac{3}{2} \int_{0}^{\infty} \frac{\mathrm{d} t}{\sqrt{(t+\alpha)(t+\delta)(t+\epsilon)^{3}}},
$$

being the elliptic integral of the second kind, $\mathscr{M}$ is the matrix whose columns are the three unit vectors $\hat{\boldsymbol{a}}, \hat{\boldsymbol{b}}$ and $\hat{\boldsymbol{c}}$ directed along the axes of the ellipsoid, and

$$
\mathscr{L}=\left(\begin{array}{ccc}
0 & -1 & 0 \\
1 & 0 & 0 \\
0 & 0 & 0
\end{array}\right)
$$

arises from the relation $\boldsymbol{u}=\mathscr{L} \nabla \psi$ between the velocity and the streamfunction in (3). The axis lengths $a, b, c$ and the unit vectors $\hat{\boldsymbol{a}}, \hat{\boldsymbol{b}}, \hat{\boldsymbol{c}}$ are determined from $\mathscr{B}$ by solving the eigenproblem

$$
\left.\begin{array}{rl}
\mathscr{B} \hat{\boldsymbol{a}} & =a^{2} \hat{\boldsymbol{a}} \\
\mathscr{B} \hat{\boldsymbol{b}} & =b^{2} \hat{\boldsymbol{b}} \\
\mathscr{B} \hat{\boldsymbol{c}} & =c^{2} \hat{\boldsymbol{c}} .
\end{array}\right\}
$$

The local background flow $\boldsymbol{u}_{b}=\mathscr{S}_{b} \boldsymbol{x}$ due to a distant 'source' vortex is obtained from a second-order Taylor series expansion of the source streamfunction $\psi_{b}(\boldsymbol{x})$ in $\boldsymbol{x} / D$-where $D$ is the distance between the centres of the two vortices-about the centre $(\boldsymbol{x}=0)$ of the 'target' ellipsoid. This gives

$$
\mathscr{S}_{b}=\gamma\left(\begin{array}{ccc}
0 & \frac{1}{2}(1+3 \cos 2 \theta)+\beta & \frac{3}{2} \sin 2 \theta \\
1-\beta & 0 & 0 \\
0 & 0 & 0
\end{array}\right),
$$

where $\gamma=\kappa_{b} / D^{3}$ is the 'strain rate', $\kappa_{b}=(4 \pi)^{-1} \iiint q_{b} \mathrm{~d} V$ is the vortex circulation (scaled by $4 \pi$ ), $\beta$ is the ratio $\left(\kappa_{b}+\kappa_{v}\right) / \kappa_{b}$ quantifying the strength ratio of the vortices, and finally $\theta$ is the angle for which the vertical separation between the vortices is $D \sin \theta$ (details may be found in McKiver \& Dritschel 2003).

Here, without loss of generality, we take $\kappa_{v}>0$. Then, when $\beta>1$, we must have $\kappa_{b}>0$ and thus $\gamma>0$, while for $\beta<1$, we must have $\kappa_{b}<0$ and thus $\gamma<0$. Hence, $\beta>1$ characterizes like-signed vortex interactions, whereas $\beta<1$ characterizes opposite-signed ones. Note that as $\beta \rightarrow 1,\left|\kappa_{b} / \kappa_{v}\right| \rightarrow \infty$, corresponding to the special cases of 'adverse' shear for $\gamma>0$ and cooperative shear for $\gamma<0$. Note also that 
two identical like-signed vortices have $\beta=2$, whereas two identical opposite-signed vortices have $\beta=0$.

The absence of vertical advection implies that the matrix element $\mathscr{B}_{3,3}$ remains constant $\left(\mathrm{d} \mathscr{B}_{3,3} / \mathrm{d} t=0\right)$. Then, considering the symmetry properties of $\mathscr{B}$, there are only five variables describing the ellipsoid in this background flow. As $\mathscr{S}_{v}$ depends on $\mathscr{B}$, the problem of finding a steady ellipsoid consists of solving the following nonlinear system of equations:

$$
\mathscr{B} \mathscr{S}^{T}+\mathscr{S} \mathscr{B}=0 .
$$

This is done numerically using an iterative linear method. From a given $n$th guess for the shape of the target ellipsoid, we calculate the corresponding velocity gradient matrix $\mathscr{S}$. Then, the linearized system corresponding to (12) is solved to evaluate $\mathscr{B}_{n+1}$, the $(n+1)$ th estimate for the steady state. The system obtained directly from (12) has a zero-determinant and thus cannot be inverted. Consequently, one equation (of the five) is suppressed and another constraint is imposed: the conservation of the volume of the ellipsoid between two iterations. Since the determinant $|\mathscr{B}|=(a b c)^{2}$, and since the volume of the ellipsoid is $\frac{4}{3} \pi a b c$, we can impose this constraint by linearizing $|\mathscr{B}|$. The volume is here set to $\frac{4}{3} \pi$ for all cases. The iterative procedure is repeated until the r.m.s. difference between the five independent coefficients of $\mathscr{B}_{n}$ and $\mathscr{B}_{n+1}$ becomes less than $10^{-10}$. Volume conservation and the absence of vertical advection ensure that the height-to-width aspect ratio $h / r$ of the ellipsoid is unchanged throughout the procedure.

Each investigated case is characterized by four parameters: the aspect ratio of the target vortex $h / r$, the strength ratio $\beta$, the angle $\theta$ and the inverse strain rate $\lambda=1 / \gamma$. For a given couple $(h / r, \beta)$, we have determined the minimum, or critical, inverse strain rate $\lambda_{c}$ for any angle $\theta$. This corresponds to the limit value of $\lambda$ below which no steady states can be found. The range of the parameter $\theta$ is restricted to $\left[0, \frac{1}{2} \pi\right]$ because of symmetry. At $\theta=\frac{1}{2} \pi$, corresponding to vortices aligned in the vertical direction, the critical inverse strain rate is always found to be zero.

In a turbulent flow, we consider the angle $\theta$ to be random. This has been verified in the simulations presented in the previous section by computing the mean value of $\theta$, denoted $\bar{\theta}$, between a given vortex and the surrounding vortex which induces the largest strain rate magnitude $|\gamma|$. For a random vortex distribution, $\bar{\theta}=\bar{\theta}_{\text {ran }} \equiv \frac{1}{2} \pi-1$ (cf. McWilliams et al. 1999). In figure 5, $\bar{\theta}$, is plotted for both like-signed and oppositesigned vortices, as a function of time (for $249 \leqslant t \leqslant 369$ ) in the $1024^{3}$ simulation. The small scatter is within the error bar estimated from the sample size, and is therefore not significant. On the other hand, in the simulation analysed by McWilliams et al. (1999), $\bar{\theta}$ for like-signed vortices was found to increase from $\bar{\theta}_{\text {ran }}$ at early times (after the first eighth of the simulation period analysed), indicating the tendency to form columnar arrays of vortices, not present in our simulations. (In fact, McWilliams et al. (1999) computed $\bar{\theta}$ from the closest vortex, not that which induces the largest strain rate. If we use the closest vortex, then for like-signed vortices we find a value of $\bar{\theta}$ significantly less than $\bar{\theta}_{\text {ran }}$. This is due to the small-scale debris that often surrounds a vortex, but which is absent in the analysis of McWilliams et al. (1999).)

Taking, therefore, $\theta$ to be random in a turbulent flow, we measure the robustness of a family of vortices, characterized by the couple $(h / r, \beta)$, by its mean inverse strain rate $\overline{\lambda_{c}}$ :

$$
\overline{\lambda_{c}}=\frac{2}{\pi} \int_{0}^{\pi / 2} \lambda_{c}(\theta) \mathrm{d} \theta .
$$

The smaller the value of $\overline{\lambda_{c}}$, the more robust the vortex is. 


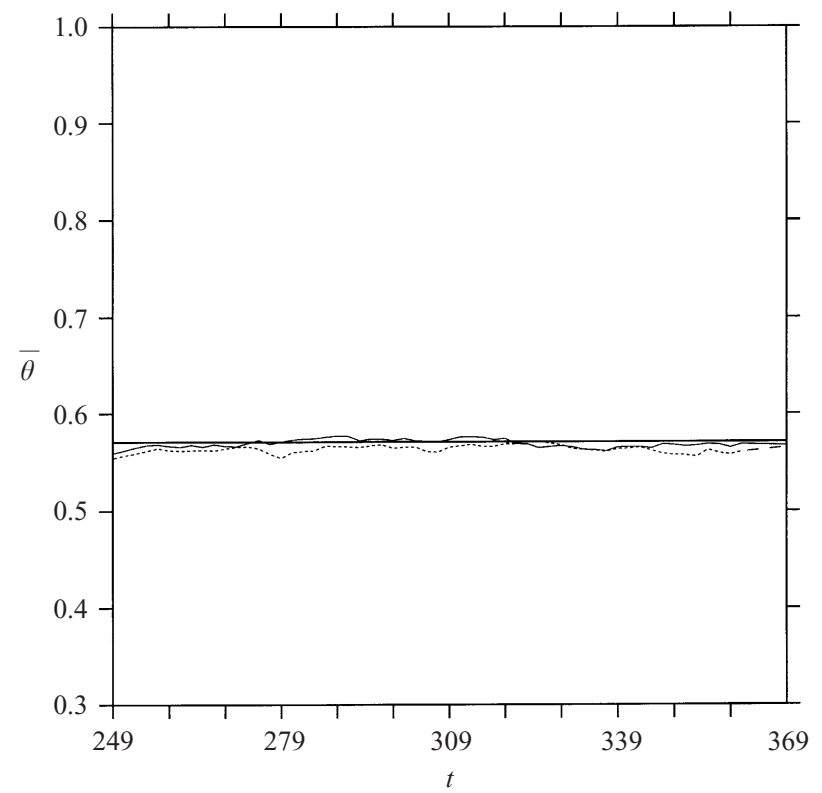

FIGURE 5. Mean angle between a 'target' vortex and the vortex inducing the highest strain on this target vortex, for ——, like-signed vortices, and ----, opposite-signed vortices, versus time in the $1024^{3}$ simulation.

\subsection{Numerical results}

We now present the numerical results. We have studied nearly 30 families of vortices of aspect ratio $h / r$ between 0.1 and 2 , with increments in $h / r$ less than or equal to 0.1 . For each aspect ratio, we have considered values of the strength ratio $\beta$ from -1.9 up to 12 . Then, for a given angle $\theta$, the calculation is started with a small strain rate $\left(\gamma= \pm 10^{-3}\right)$ and using as a first guess for the target vortex one with a circular horizontal cross-section. This choice is justified by the fact that, in the absence of background strain, a vortex with a circular cross-section is indeed a steady state. After the linear system converges on a steady state, $|\gamma|$ is slightly increased by $10^{-4}$ and the procedure is repeated until the critical value of the strain rate $\gamma_{c}$ is reached. Then, the angle $\theta$ is itself increased by $0.1^{\circ}$ until $\theta=89.9^{\circ}$.

Figure 6 illustrates the dependence of the critical strain rate $\gamma_{c}$ on the angle $\theta$ for $(a)$ equal strength vortices and $(c)$ opposite strength vortices. Figure $6(b)$ shows the results for the special case of pure shear, $\beta=1$ ( $\gamma$ is taken as positive). In all cases, $\gamma_{c}$ exhibits a singularity as $\theta \rightarrow \frac{1}{2} \pi$. The singularity is weak (integrable) in general, as seen in figures $6(a)$ and $6(c)$. However, near $\beta=1$, separating like-signed and opposite signed interactions, the singularity is stronger, as can be seen in figure $6(b)$. In this case, at $\theta=89.9^{\circ}$, the critical strain is $\gamma_{c}=47.27$. Such high values of strain are never observed in the turbulent simulations. In figure 6(c) (and for all opposite-signed vortices), there is an intermediate kink in the curve associated with a change in the orientation of the vortex; to the left of this kink, an equilibrium vortex is flattened in the direction pointing to the source vortex (with strength $\kappa_{b}$ ), whereas to the right, an equilibrium vortex is elongated in this direction. Like-signed vortices are always elongated in the direction pointing to the source vortex.

We now examine the dependence of the mean critical inverse strain rate $\overline{\lambda_{c}}$ on the aspect ratio $h / r$ for different values of $\beta$. The results are presented in figures 7 

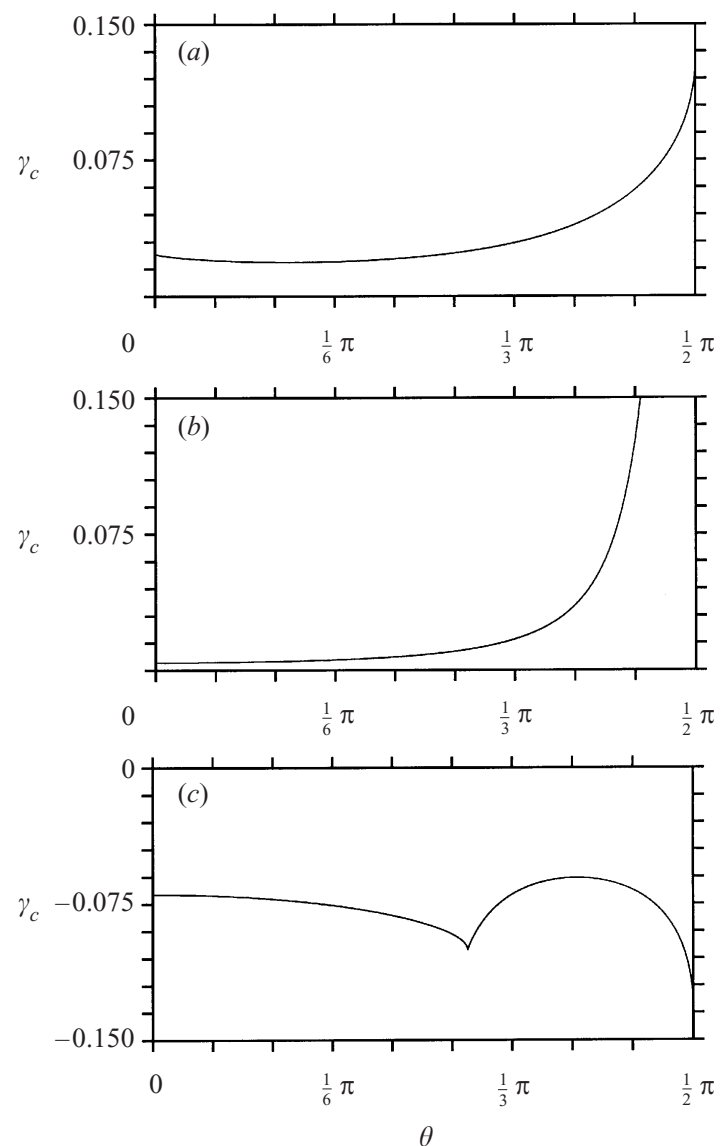

FIGURE 6. Critical strain rate $\gamma_{c}$ versus the angle $\theta$ for $h / r=1$ and $(a) \beta=2$, (b) $\beta=1,(c) \beta=0$.

and 8 for like-signed and opposite-signed interactions, respectively. (Note, in the latter $-\overline{\lambda_{c}}$ is shown since the strain is always negative.) For like-signed interactions, all the curves have a well-defined minimum at $h / r \approx 0.8$ (marked by a diamond), with a weak increase for large values of $\beta$. Large $\beta$ corresponds to a source vortex which is much smaller than the target vortex, and this kind of interaction is highly unlikely to be destructive. The relevant values of $\beta$ are $O(1)$, and for these there is little variation in the mean aspect ratio. For opposite-signed interactions (figure 8), the minimum in $-\overline{\lambda_{c}}$ occurs for $h / r \approx 1.1$, increasing to about 1.2 as $\beta \rightarrow 1$ (note: $\overline{\lambda_{c}}=0$ when $\beta=1$ in this case).

There is thus seen to be a fundamental difference between like-signed and oppositesigned interactions. Note, however, that the implied strain rate magnitudes are significantly greater for opposite-signed interactions than for like-signed ones. That is, it takes much more strain to disrupt a vortex when that strain is due to a vortex of opposite sign than when it is due to a vortex of the same sign. Therefore, if oppositesigned interactions matter in determining the favoured aspect ratio, then we would expect to see in the turbulence simulations that they are accompanied by significantly higher strain rate magnitudes. Figure 9 shows the probability density of the largest strain rate magnitudes found in like-signed and in opposite-signed interactions over the time interval $249 \leqslant t \leqslant 369$ in the $1024^{3}$ simulation. Both are strongly peaked 


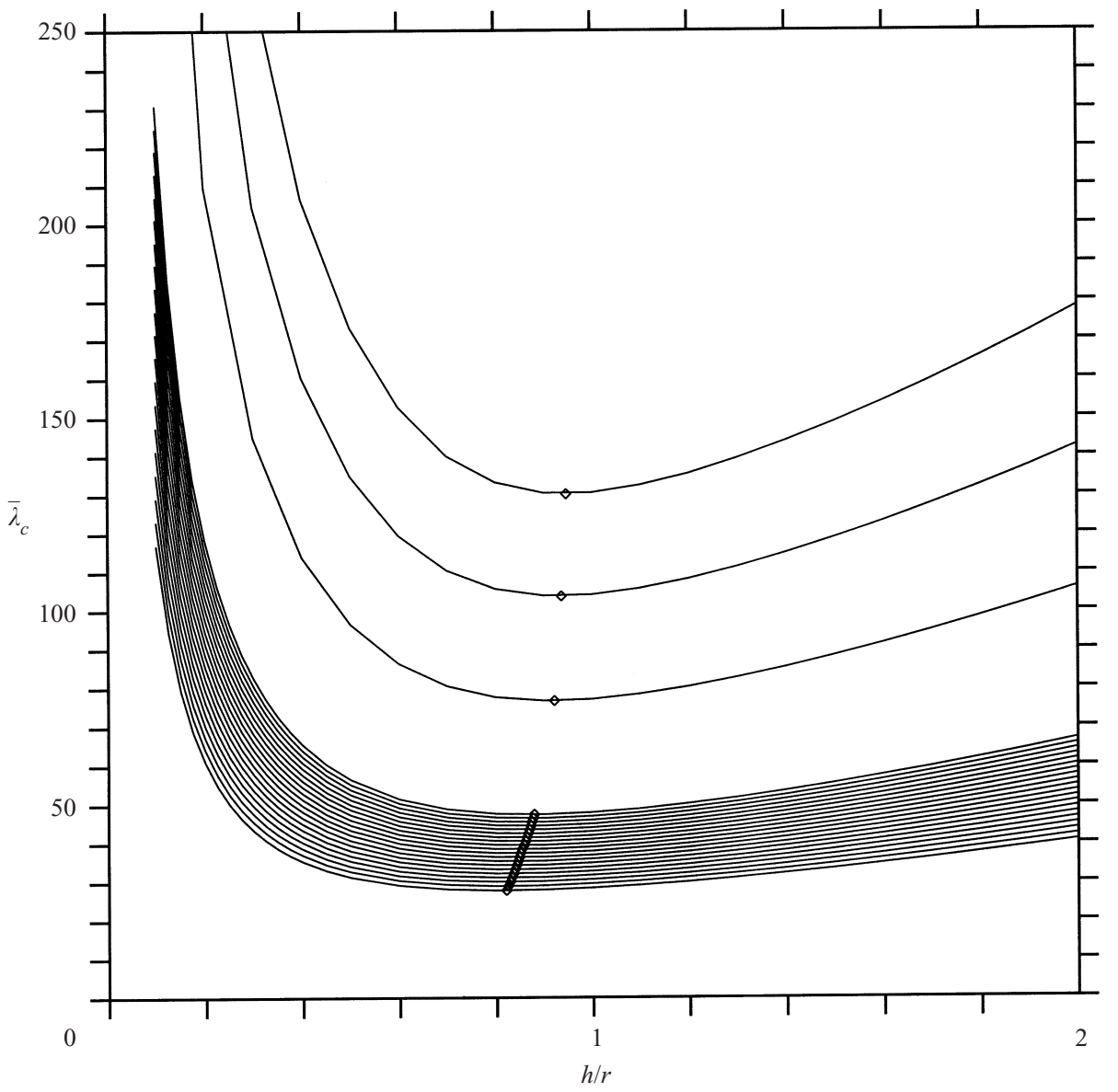

FiguRE 7. Average inverse critical strain rate $\overline{\lambda_{c}}$ versus the height-to-width aspect ratio $h / r$ for like-signed vortex interactions $\beta \geqslant 1$ (with increments $\mathrm{d} \beta=0.1$ between plotted curves except for the last three curves, corresponding to $\beta=6,9$ and 12). $\diamond$, the minimum of $\overline{\lambda_{c}}$ for each value of $\beta$, obtained from a second-order polynomial interpolation in $h / r$ around the observed minimum.

at $|\gamma| \approx 0.0046$, but are otherwise broadly similar. If anything, the largest strain rate magnitudes are found most commonly for like-signed interactions-see figure 10, which plots the ratio of the like-signed and opposite-signed probability densities. Hence, opposite-signed interactions are not important in determining the favoured aspect ratio. We have made certain of this by additionally performing simulations of opposite-signed vortices with $h / r=O(1)$ in a wide variety of configurations. In no case do the interactions result in a change in the shape of one of the vortices, even when the two vortices are initially touching.

In summary, this simple model of a steady ellipsoid predicts a favoured height-towidth aspect ratio of around 0.8 for interactions between vortices of the same sign. This is remarkably close to the value 0.8 observed in the CASL simulation results of QG turbulence, over a broad range of background flow situations. Moreover, the upturn towards $\overline{h / r}=1$ observed in figure 3 for the larger vortices, though statistically less robust, is consistent too with the large $\beta$ results in figure 7. Large vortices would frequently find themselves interacting with smaller vortices, and this interaction has large $\beta=\left(\kappa_{b}+\kappa_{v}\right) / \kappa_{b}$. Intermediate and small vortices, on the other hand, live in 


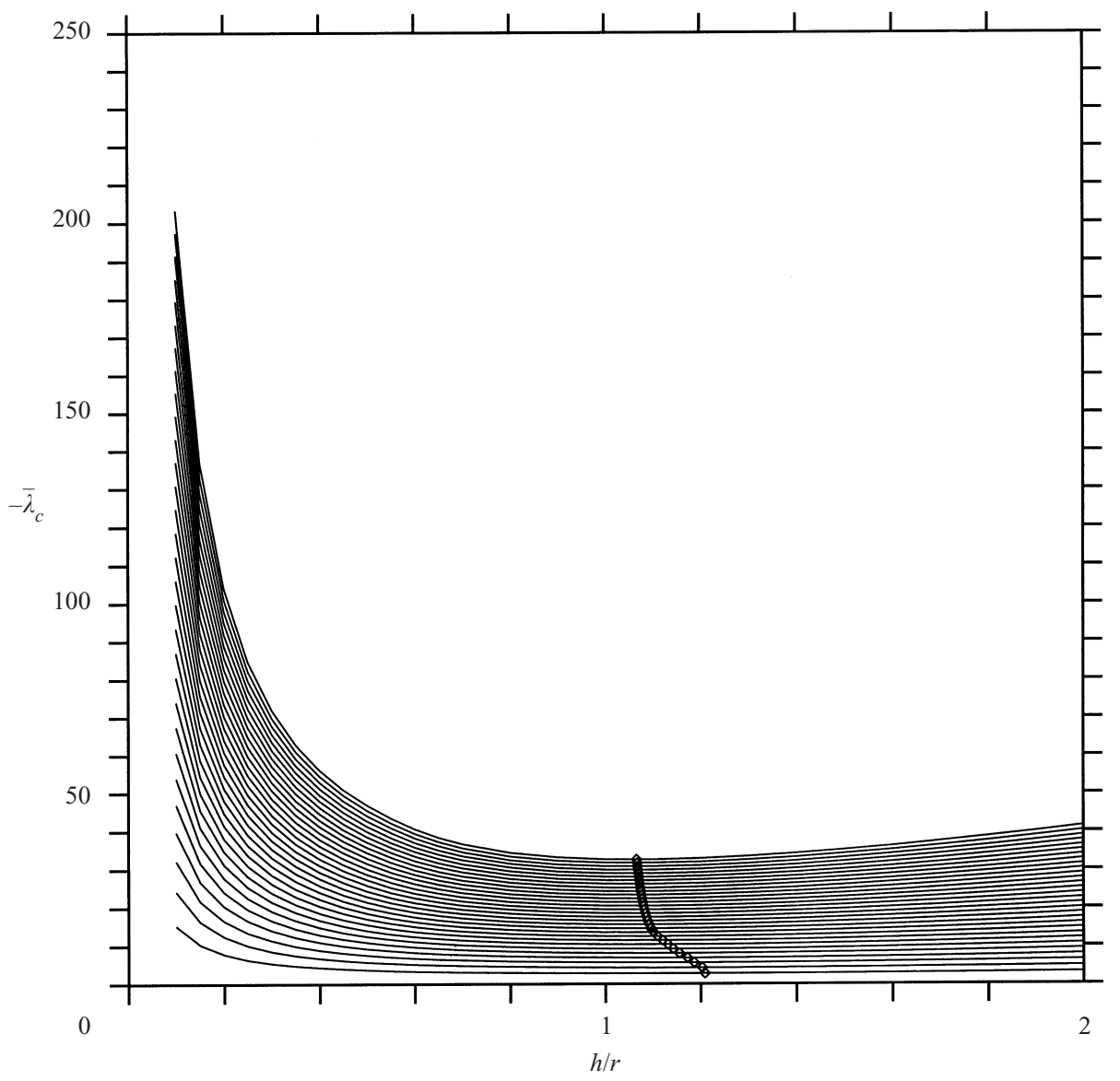

FIGURE 8 . Average inverse critical strain rate $-\overline{\lambda_{c}}$ versus the height-to-width aspect ratio $h / r$ for opposite-signed vortex interactions $\beta<1$ (with increments $\mathrm{d} \beta=0.1$ between plotted curves). $\diamond$, minimum of $-\overline{\lambda_{c}}$ for each value of $\beta$.

an environment dominated by comparable or larger vortices, and these interactions are characterized by $\beta=O(1)$, for which our simple model gives $\overline{h / r} \approx 0.8$. Indeed, when $\beta=1$, this model gives $\overline{h / r}=0.82096$. Note, finally, the steep increase of $\overline{\lambda_{c}}$ as $h / r \longrightarrow 0$. This implies that very flat vortices are unlikely to be found in QG turbulence, and this again agrees with the CASL simulations results (cf. figure 4).

\section{Conclusions}

The numerical simulation results presented indicate that the mean shape of vortices is an oblate spheroid, having a rescaled height-to-width aspect ratio of less than unity. An analysis of several highly accurate numerical simulations, conducted using the CASL algorithm at resolutions up to $1024^{3}$, implies that the mean height-to-width aspect ratio is close to $0.8 \mathrm{f} / \mathrm{N}$, and that approximately $80 \%$ of all vortices are oblate. These results were found at three different resolutions and for various time sampling periods, with very large sample sizes (exceeding 2500000 vortices in the analysis of the $1024^{3}$ simulation).

These results are further confirmed using a simple model. In this model, we 


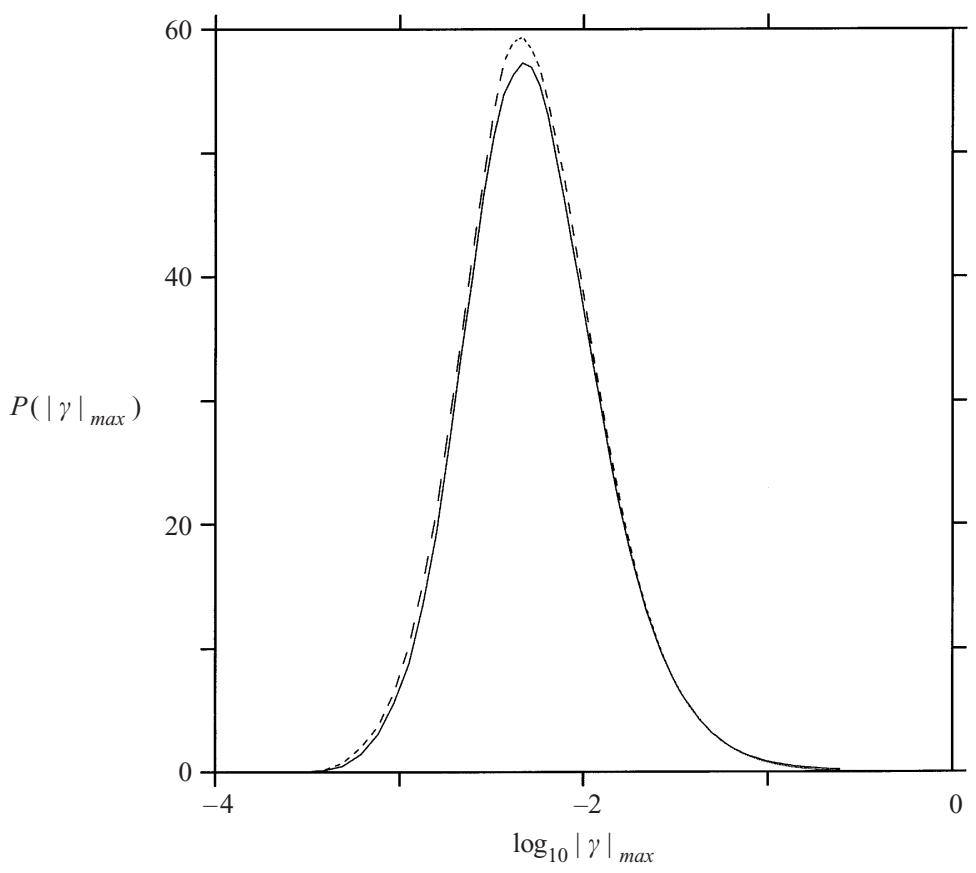

Figure 9. Probability density of the largest strain rate magnitude found in - , like-signed and - - -, opposite-signed interactions over the time interval $249 \leqslant t \leqslant 369$ in the $1024^{3}$ simulation. (Note the $\log _{10}$ scaling of $|\gamma|_{\max }$.)

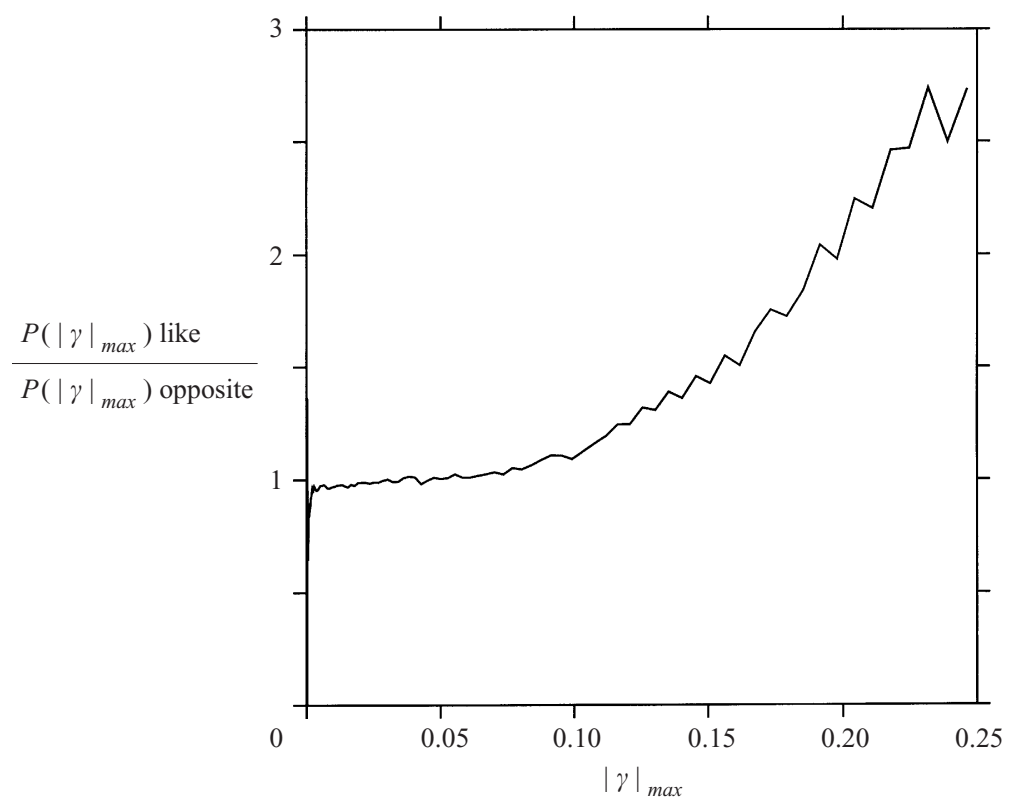

FIGURE 10. Ratio of the like-signed and opposite-signed probability densities shown in figure 9, as a function of the largest strain rate magnitude $|\gamma|_{\max }$. 
focus on the equilibrium states for a vortex embedded in a background straining flow, approximating the leading-order effects of surrounding vortices. The simple background straining flow considered is that generated by a single distant vortex arbitrarily positioned in space, and the target vortex is taken to be an ellipsoid. From a detailed analysis of all possible equilibria over a wide range of possible straining flows, we have shown that vortices with a rescaled height-to-width aspect ratio less than unity are best able to resist the highest levels of background strain. Furthermore, for small to intermediate sized vortices, the most common aspect ratio is close to $0.8 f / N$, which agrees very well with the results of the full numerical simulations. We conclude from these results, obtained in two entirely different ways, that the characteristic shape of vortices in QG turbulence is an oblate spheroid after stretching the vertical coordinate by $N / f$.

The simple model implies that there is a significant difference between the effects of horizontal and vertical shear, with the latter being a little more destructive on average. Vertical shear tends to limit the height of vortices, whereas horizontal shear tends to limit their width. Since the two types of shear are present equally in a turbulent flow, the difference between them can only be due to the lack of vertical advection in quasi-geostrophic flows. We conjecture that this difference is due to the inability of material points on the vortex to rotate vertically in response to vertical shear. On the other hand, material points can rotate horizontally in response to horizontal shear, and perhaps this freedom permits the vortex to more easily resist the effects of horizontal shear. Points at the vertical extremities of the ellipsoid can only remain there, whereas points along horizontal contours can move freely around.

The authors would like to thank Charlie Macaskill for helpful comments on the paper. J.N.R. is supported by the UK EPSRC (Grant GR/N11711). The turbulence simulations were performed on the Hitachi SR2201 at the Cambridge HPCF (for the $1024^{3}$ case) and on the CRAY T3E at CSAR in Manchester (for the $512^{3}$ and $640^{3}$ cases). C. R. K. is grateful to G. J. McMullan for ongoing help in the development of the parallel CASL software.

\section{REFERENCES}

Charney, J. 1971 Geostrophic turbulence. J. Atmos. Sci. 28, 1087-1095.

Cottet, G. H. 1996 Artificial viscosity models for vortex and particle methods. J. Comput. Phys. 127, 299-308.

Dritschel, D. G. \& Ambaum, M. H. P. 1997 A contour-advective semi-lagrangian algorithm for the simulation of fine-scale conservative fields. Q. J. R. Met. Soc. 123, 1097-1130.

Dritschel, D. G. \& MACASKILl, C. 2000 The role of boundary conditions in the simulation of rotating, stratified turbulence. Geophys. Astrophys. Fluid Dyn. 92, 233-253.

Dritschel, D. G., Polvani, L. M. \& Mohebalhojeh, A. R. $1999 a$ The contour-advective semilagrangian algorithm for the shallow water equations. Mon. Wea. Rev. 127, 1551-1565.

Dritschel, D. G. \& DE LA TORRE JuÁrez, M. 1996 The instability and breakdown of tall columnar vortices in a quasi-geostrophic fluid. J. Fluid Mech. 328, 129-160.

Dritschel, D. G., De la Torre Juárez, M. \& Ambaum, M. H. P. $1999 b$ The three-dimensional vortical nature of atmospheric and oceanic turbulent flows. Phys. Fluids 11, 1512-1520.

Gill, A. E. 1982 Atmosphere-Ocean Dynamics. Academic.

Hashimoto H., Shimonishi T. \& MiYazaKi T. 1999 Quasigeostrophic ellipsoidal vortices in a two-dimensional strain field. J. Phys. Soc. Japan 68, 3863-3880.

Koudella, C. R., Dritschel, D. G. \& McMullan, G. 2002 A parallel contour-advective semilagrangian algorithm for the integration of the quasi-geostrophic fluid model. J. Comput. Phys. (submitted). 
McKiver, W. J. \& Dritschel, D. G. 2003 The motion of a fluid ellipsoid in a general linear background flow. J. Fluid Mech. 474, 147-173.

McWilliams, J. C. 1990 The vortices of geostrophic turbulence. J. Fluid Mech. 219, 387-404.

McWilliams, J. C., Weiss, J. B. \& YavneH, I. 1994a Anisotropy and coherent structures in planetary turbulence. Science 264, 410-413.

McWilliams, J. C., Weiss, J. B. \& YavneH, I. $1994 b$ Anisotropy geophysical vortices. Chaos 4, $305-311$.

McWilliams, J. C., Weiss, J. B. \& Yavneh, I. 1999 The vortices of homogeneous geostrophic turbulence. J. Fluid Mech. 401, 1-26.

Meacham, S. P. 1992 Quasigeostrophic, ellipsoidal vortices in stratified fluid. Dyn. Atmos. Oceans 16, 189-223.

Meacham, S. P., Pankratov, K. K., Shchepetkin, A. F. \& Zhmur, V. V. 1994 The interaction of ellipsoidal vortices with background shear flows in a stratified fluid. Dyn. Atmos. Oceans 21, $167-212$.

Miyazaki, T., Furuichi, Y. \& Takahashi, N. 2001 Quasigeostrophic ellipsoidal vortex model. J. Phys. Soc. Japan 70, 1942-1953.

Miyazaki, T., Ueno, K. \& Shimonishi, T. 1999 Quasigeostrophic tilted spheroidal vortices. J. Phys. Soc. Japan 68, 2592-2601. 